\title{
The Neuroscience between Musical Stimulation and Enhancing Short Term Memory with Age
}

\author{
Maria Alexandra Fiorta ${ }^{\mathrm{a}}$, Patricia Talarczyk ${ }^{\mathrm{a}}$
}

In the field of neuroscience, cognitive memory is at the center of biomedical research. Prior works in cognitive memory all suggest and show that as people age their short term memory slows down and becomes less effective from an accuracy standpoint. This especially becomes apparent in age related memory impairments such as Alzheimer's. Using musical stimulation this study was focused on finding out if music has an effect on a musician's short term memory speed and overall accuracy in both auditory and visual memory. Visual and auditory memory are the primary ways in which music is memorized. Along with visual memory motor memory is also tested in musicians as they use muscles when playing an instrument (Chua, 2014). Thus, these two were combined with the aim of finding out if music has an effect on the entire short term memory system. The study focused on all ages ranging from fourteen to the age bracket of sixty and over. Each participant was given the same conditions under which the study was conducted. Using a T-chart analysis the data showed that overall the speed of memory is enhanced with musical stimulation; however, accuracy was statistically insignificant. To avoid the distraction of future participants they should be evaluated one at a time.

Keywords: cognitive memory, auditory, visual, musicians, motor memory

\section{Introduction}

The brain is the driving force of all processes and cognitive functions (Okano, Hirano, \& Balaban, 2000). Each memory people make over the span of their lifetimes is encoded and ingrained within their neuronal connections. These memories are what make each person different and unique. John Locke developed the philosophy that it is individual experiences and personal memories that ultimately shape a person's identity (Martin, 2000). Thus, memories are similar to a filing cabinet, storing and categorizing experiences that shape lives and individual skills. However, over time scientific studies have revealed that as the brain ages, the system that develops and stores a life's worth of memories begins to falter. The overall neuroanatomy breaks down and as a result the cognitive processes of remembering short term events slows down, and in severe cases ceases to exist. Nonetheless, research has shown that music enhances the memory and even assists in the short term memory process within older individuals. Short term memory is best described as the recollection of events or facts after a few minutes of the initial occurrence. In this paper, the relationship between age and its effects on the ability to effectively and quickly memorize a piece of music in a short period of time will be investigated. The following literary review demonstrates prior works that attempt to support the hypothesis appearing in the section titled "Gap in the Research."

\section{Literature Review \\ Introduction}

As a quasi-experimental study, this research will evaluate high school students and adults to discover if there is a relationship between age and the ability to effectively memorize music quickly and accurately in a short period of time. A review of literature was conducted to determine previous work in this field.

\section{Neuroscience of Memory}

The human brain is a complex organ responsible for all aspects of what is known as the mind (Okano, Hirano, \& Balaban, 2000). This consists of actions, thinking, feeling, learning, and memory, with memory and learning being one of the most studied processes in the field of neuroscience. Without the mind humans would be capable of nothing but simple reflexes and stereotypical behaviors (Okano, Hirano, \& Balaban, 2000). Thus, the process of developing a memory is part of a complex system within various parts of the brain, and the primary part responsible for short term memory is the hippocampus (Martini, 2012).

The memory system can be broken down into two subcategories: declarative and non-declarative memory. Declarative memory consists of more conscious memory tasks such as re-sighting an event or fact whereas, non-declarative memory does not have a conscious component and allows for skills and habits such as playing a musical instrument or riding a bike (Byrne, 1997). Thus, within this study non-declarative memory will be tested.

The Hippocampal region is the primary part of the brain responsible for short term memory. The hippocampus is concealed by the parahippocampal gyrus and dentate gyrus within the temporal lobe (Martini, 2012). This part of the brain is also a part of a complex system known as the limbic system, which is composed of the thalamus, hypothalamus, hippocampus, and amygdala (Rajmohan \& Mohandas, 2007). This system controls various functions including memory in the hippocampus, emotions in the amygdala, hormonal balances in the hypothalamus, sensory information in the thalamus, and responses to emotional stimuli (Rajmohan \& Mohandas, 2007). Memories are specifically encoded through synaptic stimulation, which leads to neuron communications allowing for a memory to be stored. Other parts used in memories are the cerebrum and cerebellum (Okano, Hirano, \& Balaban, 2000). 


\section{History of Musical Memorization}

People have been memorizing rhythms and how things sound indirectly for centuries, but the use of memorizing music to perform without a score is actually a somewhat recent development in musical performance. Prior to the nineteenth century, performing a piece from memory was actually considered arrogant. However, there is evidence that some of the greatest musical minds such as Mozart did on numerous occasions perform concerts from memory (Mishra, 2016). The mid-nineteenth century brought along a shift in what audiences hoped to see at concerts. The rise of Romanticism caused people to shift their perception of a musician as a hero. An individual was now highlighted with a romantic virtuoso (Mishra, 2016). This highlighting of a single individual out of the rest of musicians at a concert was more like modern day solos, and in many cases this led musicians to memorize the piece they were going to play to allow for improvisation to enhance their performance (Mishra, 2016).

By the 1870s, if a young musician could perform from memory a difficult repertoire, it was considered astonishing, and audiences were thrilled by such performances (Mishra, 2016). The use of memory in performances became widely respected and accepted. By 1886, whole books were being written on how to effectively memorize music. One notable example was the stepby-step manual written by W.S.B. Matthews, which highlights the use of chunking a piece into smaller phrases to allow for easier more accurate memorization (Mishra, 2016). The development of memorizing music and performing without a score is still a widely used concept today best seen in solo performances, marching bands, and jazz musicians.

\section{A Background on Music Memory}

Music memory can be defined primarily in three subcategories: auditory memory, visual memory, and motor memory (Chua, 2014). Visual memory is the primary type of music memory as this aspect involves physically looking at a piece of music and ingraining the piece into a memory (Chua, 2014). Auditory memory is also a key type of memory involved in the memorization of a piece of music, and is best defined as the ability to memorize the way something sounds. Thus, this allows individuals to internalize the sound of a piece of music with or without a score in front of them (Chua, 2014). The last focus of memory for this particular study in music is motor memory. Motor memory is memory involving movements of the body, and within music this allows players to eventually play without conscious attention to how their fingers are moving on an instrument (Chua, 2014). These aspects of memory are vital to the ability of a player being able to internalize a piece of music without the need of a score in front of them.

\section{Visual Memory}

Visual memory is an important skill when memorizing a piece of music as this is the first step taken at memorizing a piece. What the person sees on the page translates into something they can simply recall later once encoded. This task is somewhat like memorizing words, but slightly more challenging seeing that what is presented on the page is simply black dots and musical markings. In a study regarding memory, participants were tested on their ability to memorize and recall a set of words, which were categorized by negative and positive biases (Saarela et al., 2017). This was testing visual memory with an approach of looking at whether or not an emotional stimuli could result in better memorization. Similarly, music can have an emotional effect based on the way it sounds; however, the visual component does not include emotions. Visual music memorization is testing the ability to remember as many details as are presented to the individual, thus, this aspect of music memory is looking more at accuracy of what one sees more so than anything else (Cheung, Chan, Liu, Law, \& Wong, 2017).

\section{Auditory Memory and Motor Memory}

Auditory memory plays a vital role in the memorization of music. There is research to suggest that when a child is born they are already capable to recognizing certain sounds, particularly their mother's voice, and as they grow this skill develops even further. The use of this skill allows musicians to remember the way a piece sounds and internalize the music. Research shows that individuals with training in music show signs of stronger neuronal activation in the hippocampus, frontal, and temporal cortices during an auditory task (Cheung, Chan, Liu, Law, \& Wong, 2017). In addition, musically trained individuals also display increased advantages in tasks including short-term auditory learning and auditory memory capacity for complex auditory patterns (Cheung, Chan, Liu, Law, \& Wong, 2017). A greater music background also shows better auditory memory performance for a longer period of time (Cheung, Chan, Liu, Law, \& Wong, 2017).

There is also growing cognitive research to suggest that there is a relationship between skills such as playing an instrument and connections between auditory and motor memory along with motor memory enhancing links between perception, memory, and action (Mathias, Tillmann, \& Palmer, 2016). For individuals who have experienced playing a musical instrument hearing musical sounds can trigger cortical motor regions (Brown et al., 2013; Lahav, Saltzman, \& Schlaug, 2007; Haslinger et al., 2005). Therefore, when people learn melodies through auditory-memory production they are able to recognize that melody better (Brown \& Palmer, 2012). This fact means that the effect of motor learning on memorizing music suggests that previously performed music recognition differs from that of something that was only ever heard before. Thus, there is a connection between auditory and motor memory skills, which when practiced together enhances the overall effectiveness of what an individual remembers (Mathias, Tillmann, \& Palmer, 2016).

\section{Gap In the Research}

This study will attempt to answer the following question: How is age related to the ability to effectively memorize music? The research will address a gap between age and the effects musical stimulation has on the memory of individuals when tasked with memorizing a piece of music through auditory and visual memory. The study will look at the overall speed and precision of varying ages to see if age does slow the process down or simply make it less accurate. It is hypothesized that age will not have an 
effect on an individual's overall ability to memorize a piece of music effectively; however, age will cause the memorization process to be slower in older participants. This will also address the process of music therapy in individuals with memory impairments such as Alzheimer's. The question of whether patients should also play the musical instrument along with the therapists will be investigated. The data will also attempt to look into the gap behind if musicians tend to have overall better short term memory recall even with age.

\section{Methods}

The research found in this paper was conducted through the use of a quasi-experimental study. The study was aimed at finding the relationship between musical stimulation increasing short term memory as one ages through attempts to effectively memorize a short excerpt of music through visual and auditory methods.

For this particular study only one method was used similarly to that of another research study. The auditory memory test used was taken from a study that conducted research on sensory, cognitive, and sensorimotor learning and its effects on music recognition (Mathias, Tillmann, \& Palmer, 2016). This auditory memory portion of the study consisted of two parts. Participants first listened to a short, eight- measure excerpt of music, which was played with differences from the original score. Then after three minutes, which is the specific amount of time for short term memory, the participant listened to the same piece, but now played correctly. They were given the original score in this second round, and as they listened they were given the task of circling as many of the errors from the first round as they could remember. There were a total of three errors. One was a rhythm played differently, and the other two were pitches that were changed to be natural and sharp. They then would receive a score of zero to three depending on how many they correctly identified, and this score later factored into their overall score.

The visual memory portion of the study focused primarily on remembering what one saw on the page. The participant was given thirteen measures of an excerpt of moderately difficult music. The music given to participants can be seen in Appendix A. They then were given twenty allotted minutes to memorize as much of the piece as possible with as close to one hundred percent accuracy as possible. Five-minute increment checks were made to see the progress of the participant. However, the participant could choose to play from memory at any given time so long as they felt ready. The idea was to play as much as possible in the shortest period of time. When the participants were ready they were recorded, which could later be analyzed for the accuracy of notes and rhythms, the amount of time used, the amount of measures played, and the tempo at which they played. The rubric used to assess each participant had a possible total score of seventy five, which consisted of seventy two possible points for the visual memory portion plus an additional three points for the auditory test. This rubric can be viewed in appendix B. The idea was to receive as many points as possible, which would show greater short term memory with musical stimulation.

There was no manipulation from participant to participant. Each person received the same time, music, and study settings, which consisted of two people in one room on opposite ends of the room. Participants were instructed that they were not permitted to assist each other, but were allowed to implement any method they felt would help them memorize the music in the most accurate way. Observations were made in regards to how participants chose to memorize their music.

\section{Participant Characteristics}

There were thirty two total participants in this study. Sixteen of the participants were in the adult age group ranging from twenty three to sixty six. The other sixteen participants were high school age, which ranged from fourteen to eighteen. Thus, basically any age was allowed to participate from fourteen up. Participants had to have had musical education, and they had to have been playing their instrument for at least one year prior to the study. Experience was not a variable in this study, but participants were expected to be able to sight read and memorize the piece on their own, thus, being able to play an instrument effectively was a requirement.

\section{Sampling Procedures}

There were two locations in this study. The location for high school students was a local high school, and the adult age group was conducted at a local middle school. However, the settings of the data collection remained the same for each participant. Two participants were in one room. They were not allowed to assist each other, thus, they were situated on opposite sides of the room. The rooms used were small practice rooms, and participants were given a piece of music marked with stopping and starting points, a stand, a writing utensil, and a chair. The only system used to choose participants was that they were expected to play a musical instrument that is considered a band wind instrument. Thus, no string instruments, pianos, or percussion instruments were allowed in this study. The only other requirement was that the participant had to be at least fourteen years of age.

\section{Measures and Covariates}

The variables that were measured within this study were primarily five things: accuracy of visual memory notes and rhythms, accuracy of auditory memory identification, amount of measures memorized, tempo accuracy, and age. The instruments used to collect this data were primarily a stopwatch to time each participant, a recording device used to later analyze the participant's performance, and paper copies of the music.

\section{Research Design}

Participants within this study were observed slightly manipulated, but mostly natural. The only manipulated portion of the study was that they had a time limit, and that they had another person in the room participating at the same time. However, overall participants were observed naturally because the study was looking at their individual short term memory. Each 
participant's memory has a slightly different capacity, so the only way to obtain the most accurate results was to observe them naturally. If a participant was unable to play all thirteen measures from memory that was perfectly acceptable. Consequently, they would receive a lower score; however, participant's naturally playing as much as possible was important to obtaining accurate data. In addition, the participant chose when they were ready to recorded completely from memory. Thus, some naturally would do better than others. Memory is best measured naturally similar to the way a test shows how much a student can remember. This study aimed to test how much people of various ages could remember from what they saw and heard. Voluntary consent was given to ensure that participants knew they could opt out of the study at any given time. In addition, there were no incentives for participation. Thus, participants knew they would be required to try and memorize as much as naturally possible.

\section{Results}

The results from this study examined three specific types of quantitative data to determine statistical significance. The first statistical analysis looked into the connection between age and the speed of the participant's memory. This portion of the memory test involved analyzing the specific part of the proposed research question of if musical stimulation enhanced memory with age. The second analysis was between age and auditory memory. The last analysis was the connection between visual memory accuracy with age, so this was the second largest portion of the proposed research question as this portion studied the overall accuracy of memory with age during musical stimulation. The variables of age, speed, auditory memory, and visual memory accuracy were analyzed to determine whether musical stimulation enhanced the speed and accuracy of short term memory.

\section{Visual Memory Test}

The largest variable being tested was the speed of memory with age as most prior studies have shown that the speed of memory formation slows down as an individual gets older. In this study the data collected for the connection between age and speed of memory was significant. To analyze this data an independent T-test was performed (Stangroom, 2018). The T-test showed that there was significance at $\mathrm{p}<.05$, with the $\mathrm{p}$ value being equal to .002849 (Stangroom, 2018). The data also showed that the adult age group was about four minutes faster on average than the younger age group with 90 percent confidence. This data can be viewed in figure one below. The graph shows that the older age groups did in fact use less time than their younger counterparts. However, the accuracy was sacrificed at the speed as the values tested in the T-test between age and visual memory accuracy were not significant at $\mathrm{p}<.05$ (Stangroom, 2018). The $\mathrm{p}$ value for this test was .136335. Now despite the insignificance of age with visual memory accuracy the test did show that the adult age group did on average have a slightly higher score by about 2.44 points more than the younger age group.

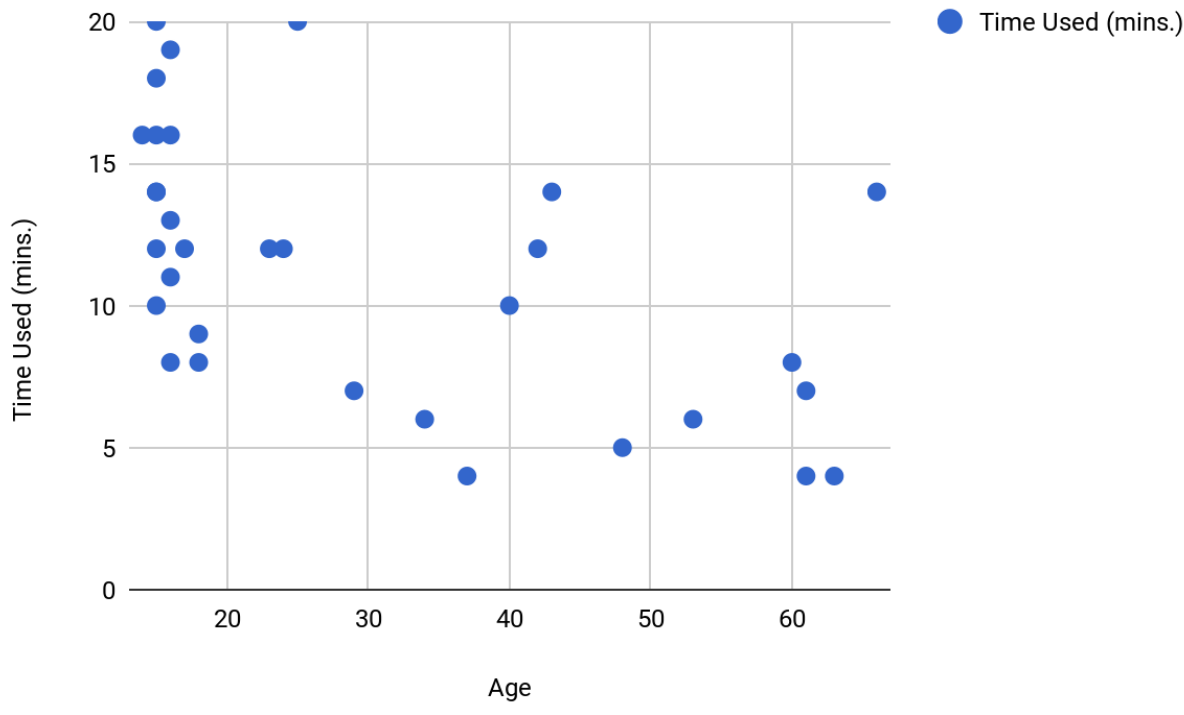

Figure 1. Time Used vs. Age

\section{Auditory Memory Test}

Auditory memory was also analyzed through the use of an independent T-test (Stangroom, 2018). This particular test was aimed at seeing if musical stimulation would also enhance auditory memory. However, this test also came out to be not statistically significant at $\mathrm{p}<.05$ with a $\mathrm{p}$ value of .261437 (Stangroom, 2018). Once again despite the nonstatistical significance of the test, the results did show that the adult age group did have a higher average score on this portion of the study as opposed to the younger age group by about .19 points more. Thus, although the statistical analysis showed that musical stimulation did enhance the overall speed of the older participants' memory, there was no significance with the other results. However, the averages showed that the adults did tend to score slightly higher from an accuracy standpoint than the younger participants. In figure two below the results of all three specific data variables are shown. 


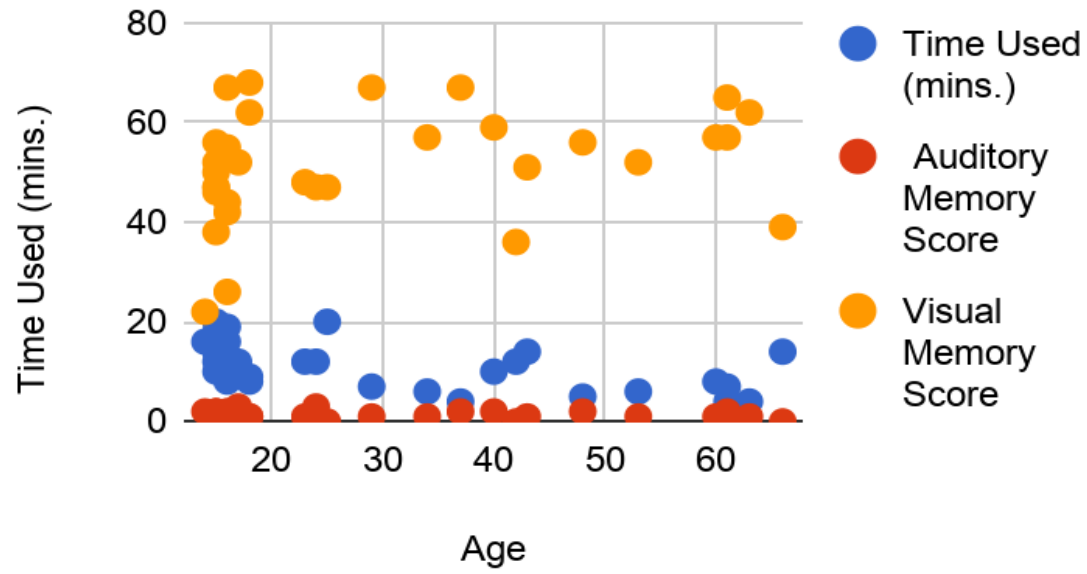

Figure 2. Time Used vs. Memory Scores

Analysis

The statistical analysis showed significance in that the older participants were faster than the younger participants. As previously mentioned, the adults were about four minutes faster than the younger participants on average. This means that the adults in the visual memory portion of the study used fewer of the 20 allotted minutes when attempting to accurately memorize as much of the excerpt of music provided as possible. Thus, with musical stimulation their short term memory speed increased whereas the younger individuals were slower. The argument can be made that the older participant's synaptic neuron responses in the Hippocampal region were faster when creating short term memory connections as opposed to the younger participants. This is significantly different than the previous idea that age slows down the memory, showing that music does indeed enhance the speed of memory in older individuals.

\section{Short Term Memory Accuracy}

Although this study showed enhanced short term memory speed in older individuals the overall accuracy of the music memorized was not statistically significant. Thus, while the adults did on average score slightly higher than the younger individuals the scores with age were not statistically significant due to the fact that the scores in both age groups ranged from near perfect to virtually no recollection of the piece of music. This means that while speed was enhanced accuracy was not, and this does correlate with prior works as other studies have shown music to trigger a long term memory response, but not necessarily short term accuracy in older individuals. This is especially evident in older individuals with memory impairments such as Alzheimer's. Long term memory differs from short term memory because long term memories are engrained in neuronal connections that have been present for a long period of time, whereas short term memories occur within a short time frame. Thus, music tends to enhance memory accuracy by assisting older individuals in remembering an event, for instance, that occurred longer ago. However, music has little effect on short term memory accuracy.

\section{Observations}

Observations were made in regards to how participants first chose to memorize once given the music. Most participants immediately began to play their instruments and worked through the sections they had a hard time with. They then would repeat those harder sections over and over again until they felt confident with those measures. However, some participants chose to first finger along through the music without playing a single note until they chose to record as much as they could from memory. This method was interesting because participants were more or less using motor memory over the visual component. When an instrument is played there is always a level of motor memory involved in remembering what it felt like to play a note or rhythm; however, this motor memory portion is exemplified when a musician simply does not play and only works out the fingerings. This is because the musician is then remembering how it feels to play something without every truly playing the music. Thus, the memory of these participants was not in the brain rather in the muscles used to play their respective instruments. Most of the younger participants first fingered through the music and then played, whereas, their adult counterparts tended to simply begin playing. This could have had an effect on the results of the study as different participants were using different methods in which to memorize the excerpt presented to them. Therefore, they were essentially using different aspects of the memory system when initially attempting to memorize the music.

\section{Auditory Memory Accuracy}

The results of the auditory memory were interesting as other studies have shown that with auditory stimulation short term memory recall was better in older individuals. However, within this particular study the auditory memory test results were not 
statistically significant. The older participants did have slightly higher scores than the younger participants, but due to the fact that data within each group did not have a trend and was significantly spread out, the results hold no real significance. Thus, this study showed no significant auditory memory recollection improvement with musical stimulation. A possible error in this portion of the study that may have resulted in the non statistically significant data was the fact that the environment participants were listening to the excerpt in was not always quiet as the data was collected in the music wings of school buildings. Therefore, at times the participants may have had a difficult time hearing what was being played for them, which may have affected their ability to accurately memorize and recall what they heard.

\section{Overview}

Overall the older group of participants did do better than the younger group. The older participants were much faster in memory recall and were more accurate. However, there were aspects of the study that show little accuracy improvement in the memory system of individuals from fourteen to sixty six. The first aspect of this was the component of visual accuracy in a short period of time. Neither group benefited overall from musical stimulation being combined with visual memory from the standpoint of accuracy. In addition, the adults and younger participants also did not have better short term memory recall auditorily with musical stimulation. Thus, the only enhancement the music had on the memory system was from the standpoint of speed. Therefore, the hypothesis was incorrect. Initially the study hypothesized that age would not affect the ability to effectively memorize music. Also that age would affect speed causing older participants to be slower. However, the results show that the opposite occurred. The older participants were faster, but accuracy was not affected in either age group with musical stimulation.

\section{Conclusion}

In conclusion, the effects music had on short term memory were mostly effective when looking at the overall speed of both age groups. The accuracy of the visual memory was not necessarily enhanced, but when looking at the future implications of the results, this study has shown that with some form of musical stimulation,short term memory speed does improve significantly, especially in older individuals. This means that the assumption can be made that through the use of musical stimulation adults can create short term memories at a much faster rate than previously believed.

\section{Limitations}

Some limitations that may have led to the auditory memory test and the visual accuracy scores not being statistically significant may have been due to lack of clarity in directions. For future studies participants should be told that accuracy should not be sacrificed for speed. Many of the participants in both age groups tended to rush through things when attempting to memorize the excerpt of music just so they could have a faster time. While the idea is to use the least amount of time, the study was also aiming at visual memory accuracy naturally, so the hope was for participants to play as much as possible with the best accuracy as possible in the shortest period of time. However, what ended up happening was that many individuals would simply only memorize a few measures as opposed to attempting to memorize the whole excerpt in efforts to have a faster time.

Another limitation was having two participants present in the room at the same time while conducting the study. As a result there was a slight lack of focus in many of the participants because they would become distracted from the other participant's playing. Thus, this may have had an effect on the overall visual memory score of each participant due to their distraction. The other limitation was the noisy environment. The rooms containing the participants were located in a very noisy part of the building. This may have made the auditory memory portions difficult to hear, thus affecting their overall ability to recall what they heard. Thus, for future studies the room should be in a quiet environment with one participant in the room at a time.

\section{Implications}

The future implication of this study is that musical stimulation did enhance the overall speed of the adult participants. While the short term memory accuracy was not affected, the speed being enhanced is significant as older individuals usually are believed to have slower short term memory speed. This means that through the use of musical stimulation in the brain, older individuals are able to make connections for memory faster than even the younger group. Therefore, when treating older patients with memory deficiencies or impairments, musical stimulation could possibly assist them in developing short term memories at a faster rate than before. In addition, this holds future implications for music therapists who could potentially assist their patients by not only playing the instruments for them, but also allowing their patients to play along. This would continue to enhance the stimulation of their short term memory system by causing them to overtime be faster with the formation of short term memories. The results of this study also indicate that allowing older patients to play an instrument even as simple as hitting a drum could potentially have positive implications on the speed of their short term memory system. Further research would need to be conducted in order to see if accuracy is negatively impacted by the increased speed.

\section{References}

Brown, R. M., \& Palmer, C. (2012). Auditory-motor learning influences auditory memory for music. Memory \& Cognition, 40, $567-578$.

Byrne, John H. "Learning and Memory ." Learning and Memory (Section 4, Chapter 7)Neuroscience Online: An Electronic Textbook for the Neurosciences | Department of Neurobiology and Anatomy - The University of Texas Medical School at Houston, nba.uth.tmc.edu/neuroscience/m/s4/chapter07.html.

Cheung M-c, Chan AS, Liu Y, Law D, Wong CWY (2017) Music training is associated with cortical synchronization reflected in 
EEG coherence during verbal memory encoding. PLoS ONE 12(3): e0174906. https://doi.org/10.1371/ journal.pone.0174906

Chua, W. L. (2014, March \& april). A closer look at strategies for memorization[Music Memorization].

Haslinger, B., Erhard, P., Altenmüller, E., Schroeder, U., Boecker, H., \& Ceballos-Baumann, A. O. (2005). Transmodal sensorimotor networks during action observation in professional pianists. Journal of Cognitive Neuroscience, 17, 282293.

Lahav, A., Saltzman, E., \& Schlaug, G. (2007). Action representation of sound: Audiomotor recognition network while listening to newly acquired actions. Journal of Neuroscience, 27, 308-314.

Martini, Frederic H., et al. Human Anatomy, Seventh Edition

Martin, R. (2000). Locke's psychology of personal identity. Journal Of The History Of Philosophy, 38(1), 41.

Mathias, B., Tillmann, B., \& Palmer, C. (2016). Sensory, Cognitive, and Sensorimotor Learning Effects in Recognition Memory for Music. Journal Of Cognitive Neuroscience, 28(8), 1111-1126. doi:10.1162/jocn_a_00958

Mishra, J. (2016). PLAYING FROM MEMORY. American Music Teacher, 65(6), 12-16

Okano, Hideyuki, et al. "Learning and Memory." Proceedings of the National Academy of Sciences of the United States of America, 17 Oct. 2000, web.a.ebscohost.com/ehost/pdfviewer/pdfviewer?vid.

Rajmohan, V., \& Mohandas, E. (2007). The limbic system. Indian Journal of Psychiatry, 49(2), 132-139. http://doi.org/10.4103/0019-5545.33264

Saarela C, Joutsa J, Laine M, Parkkola R, Rinne JO, Karrasch M (2017) Regional gray matter correlates of memory for emotionladen words in middle-aged and older adults: A voxel-based morphometry study. PLoS ONE 12(8): e0182541. https://doi.org/10.1371/journal.pone.0182541

Stangroom, J. (2018). Social Science Statistics. Retrieved from http://www.socscistatistics.com/Default.aspx

This study was completed while the author was at Mentor High School as an AP Research student. The author would like to thank all of the individuals who participated in this study. There were no conflict of interests in this study.

\section{Appendix A}

\section{Trumpet Bb}

\section{Long, Long Ago}
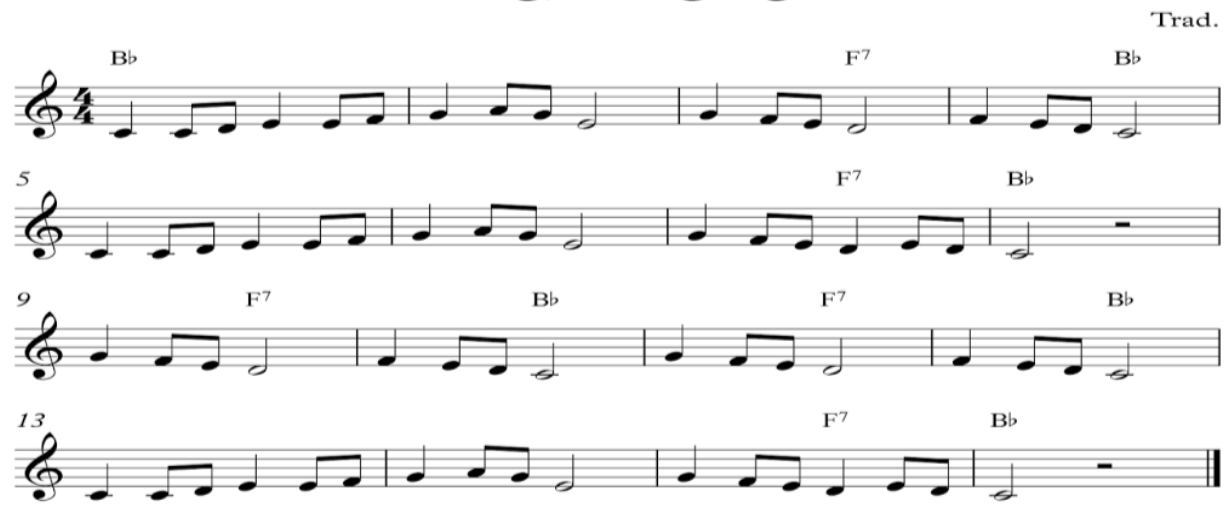

Free trumpet sheet music at Capotasto Music

\section{Appendix B}

Time Taken (out of 20 minutes)

$16-20$ minutes $=5$ points $(25 \%)$

$11-15$ minutes $=10$ points $(50 \%)$

$6-10$ minutes $=15$ points $(75 \%)$

5 or less minutes $=20$ points $(100 \%)$

\section{Accuracy from Memory}

Notes/rhythms completely wrong $=5$ points $(25 \%)$

Notes/rhythms multiple errors that make it indistinguishable $=10$ points $(50 \%)$ 
Notes/rhythms with few errors but music still understandable $=15$ points $(75 \%)$

Notes/rhythms with few to no errors $=20$ points $(100 \%)$

Suggested Tempo Accuracy

Slower $=10$ points $(50 \%)$

Close to accurate $=20$ points $(100 \%)$

Number of Measures Played

$12-13=12$ points

$10-11=10$ points

$8-9=8$ points

$6-7=6$ points

4-5 = four points

2- $3=2$ points

Auditory Memory Test

1 Correct $=1$ points

2 Correct $=2$ points

3 Correct $=3$ points 